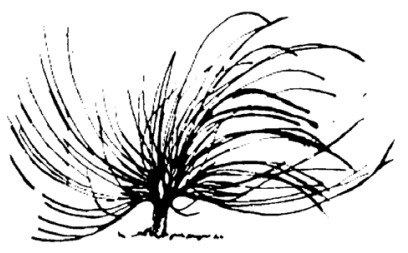

\title{
Mundo de la vida, habitus y reproducción en el análisis de la segmentación social de la educación chilena
}

\author{
Alejandro Sánchez Oñatel \\ Universidad de Concepción \\ Chile \\ asanchezo@udec.cl
}

\begin{abstract}
Resumen
Este ensayo ofrece una revisión conceptual de los aportes de la sociología en la comprensión del fenómeno de la segmentación social de la educación, particularmente a partir del caso del sistema educativo chileno. En ella se revisa el concepto de mundo de la vida aportado desde la sociofenomenología de Alfred Schütz y los conceptos de habitus y reproducción incorporados por Pierre Bourdieu, en relación con antecedentes empíricos del contexto educacional chileno que muestran la expresión de la segmentación y otras formas de desigualdad en la educación escolar y universitaria.
\end{abstract}

Palabras clave: educación, sistema educativo, segregación escolar.

\begin{abstract}
This paper provides a conceptual review of the contributions of sociology in understanding the social segmentation of education, particularly from the case of the Chilean education system. Here, with the lifeworld of social
\end{abstract}

\section{(ब) $(1 \otimes \Theta$}

Recibido: 21 de agosto de 2018. Aprobado: 12 de setiembre de 2019.

http://dx.doi.org/10.15359/rep.14-2.1

1 Psicólogo (Universidad del Desarrollo), Magíster en Investigación Social y Desarrollo. 
phenomenology provided by Alfred Schutz and the concepts of habitus and reproduction incorporated by Pierre Bourdieu, empirical data regarding the Chilean educational context showing expressions of segmentation and other forms of inequality in school and university education are reviewed.

Keywords: education, educational systems, school segregation

...y no fue tan verdad, porque esos juegos al final terminaron para otros con laureles y futuro y dejaron a mis amigos pateando piedras.

(El Baile de los que sobran, Los Prisioneros)

\section{Introducción}

$\mathrm{U}$

na de las características centrales del sistema educacional chileno es la lógica mercantil, que permite sostener una clara segmentación socioeconómica de los estudiantes, con altos niveles de polarización en los establecimientos; lo cual ha sido discutido tanto en el nivel nacional como internacional. Así, las distintas dependencias administrativas que configuran el sistema, mediante la agrupación de estudiantes, según su nivel socioeconómico y características culturales, afectan la distribución y la calidad de los resultados de aprendizaje de la educación en general (Villalobos y Valenzuela, 2012; García-Huidobro, 2013).

Basado en este supuesto, este trabajo busca analizar estas características definitorias del sistema educacional chileno, a la luz de los aportes de la sociología, particularmente, desde las contribuciones de la sociofenomenología de Alfred Schütz y la sociología contemporánea de Pierre Bourdieu, facilitando así la comprensión de elementos como la segmentación social, la selección y la evaluación, propios del sistema educativo chileno en el nivel escolar y universitario.

\section{Un análisis de la segmentación social de la educación}

\section{El mundo de la vida en la sociología fenomenológica}

La sociofenomenología se basa en la filosofía de Husserl y en el método de comprensión (versehen) de Max Weber. El debate tiene una 
vertiente epistemológica, sobre cómo lograr el conocimiento, y una vertiente metodológica, respecto de cómo aproximarse a una comprensión fenomenológica de lo cotidiano (Rizo, 2006).

Desde el punto de vista epistemológico, la sociofenomenología implica romper con las formas de pensamiento de la sociología tradicional, enfatizando la necesidad de comprender la realidad en el durante (aquí y ahora). Este programa teórico comienza desde la interpretación de la realidad a través de los significados subjetivos que otorgan a ella los sujetos sociales. En otras palabras, su estudio se centra en la forma en que las estructuras de la realidad se constituyen objetivamente y se instituyen intersubjetivamente, como reconstrucción permanente de la vida social (Rizo, 2006; 2014).

De acuerdo con Schütz ${ }^{2}$ (1970), existen algunas estructuras fundamentales del mundo de la vida, que son aceptadas sin cuestionamiento alguno. Estas estructuras son heterogéneas, en el sentido de que nuestro conocimiento y nuestra capacidad para actuar sobre ellas exhiben múltiples estratificaciones.

Preliminarmente, es posible distinguir entre un mundo de alcance real, del cual el sujeto tiene una percepción directa, sin estar mediada por instrumentos que la faciliten; y un mundo que antes era, que ya no es, pero que en principio es posible traerlo al alcance real, aceptando sin cuestionamientos que el estado restaurado de este mundo es tal cual el anteriormente experimentado, aunque puedan admitirse pequeñas modificaciones. En cualquier caso, la experiencia del ámbito social se remitirá siempre a la situación biográfica de cada sujeto. Esta experiencia se denomina social directa, y es compartida con sujetos semejantes en un lapso y en un margen del mundo espacial comunes. Por otra parte, tenemos la posibilidad de actuar sobre el mundo de nuestros sucesores, el cual no influye sobre nosotros; pero no podemos actuar en el mundo de nuestros predecesores, el cual sí influye en el que vivimos (Schütz, 1970).

Por lo tanto, nuestra experiencia social estará predefinida o condicionada por la interpretación y la expresión que prevalezca dentro del grupo social al que pertenezcamos, y corresponderá a lo que acostumbramos a llamar cultura del grupo, la cual aceptamos sin

2 Filósofo y sociólogo austríaco (1899-1959). Cofundador de la International Phenomenological Society. Discípulo de Husserl, Schütz, intentó una fundamentación fenomenológica de las ciencias sociales, buscando descubrir las estructuras en que el mundo de la subjetividad, o mundo social, se constituye en la experiencia cotidiana (Fuente: www.menbiografias.com). 
cuestionamiento (sin perjuicio de que sea efectivamente cuestionable -y digna de cuestionar-), ya que suele determinar, también el tipo de problemas sociales que pueden ser considerados resueltos dentro de los márgenes de explicación cultural (Schütz, 1970).

Todo esto permite dilucidar que una muy pequeña medida del conocimiento de cada individuo se origina a partir de su experiencia personal. La inmensa mayoría del conocimiento es socialmente derivada y transmitida hacia el sujeto a lo largo de su proceso educativo, ya sea desde la familia, la escuela, y todo tipo de relaciones sociales. Este conocimiento puede abarcar desde cosmovisiones y creencias más o menos fundadas, máximas y premisas, hasta instrucciones y recetas para resolver problemas sencillos y cotidianos; de tal manera que todo el conocimiento sobre el mundo de la vida queda validado, articulado y probado por el grupo sociocultural (Schütz, 1970). Un ejemplo muy prototípico de un mecanismo de transmisión de conocimiento del grupo cultural dominante es el currículum escolar, que define los campos de aprendizaje y contenidos mínimos y obligatorios que todo individuo que ingresa al sistema educativo escolar debiese conocer al momento de egresar de este.

Cabe señalar también que estas formas de conocimiento concerniente al mundo de la vida, se diferencian de múltiples maneras y pueden variar para el individuo, de individuo a individuo, de individuo a grupo social, para el propio grupo y, finalmente, de un grupo a otro. El contenido de lo que se conoce y de lo desconocido es, en efecto, relativo: para el individuo en relación con su situación biográfica, y para el grupo en relación con su situación histórica (Schütz, 1970). Continuando con el mismo ejemplo, dos estudiantes de un mismo establecimiento han de adquirir parcialmente los mismos conocimientos, con variaciones que dependerán de la demás influencia sociocultural derivada de su pertenencia a otros grupos sociales (por ejemplo, familiar, comunitario, religioso). Asimismo, dos estudiantes de un mismo establecimiento que hayan sido educados en distintas generaciones, o bien, dos estudiantes de establecimientos de distinta dependencia administrativa (público o privado) dada su situación histórica, habrán de adquirir un conocimiento del mundo social relativamente diferenciado uno del otro.

Es así que, siguiendo a Schütz (1970), una categoría fundamental de la vida social es la desigualdad de la distribución del conocimiento en sus diversas formas. Ante esta premisa, el autor se pregunta ¿qué es 
lo que determina esta diferenciación? La respuesta estará en el hecho de que cada sujeto experimenta su estructura fundamental del mundo de la vida como un correlato subjetivo de las diversas formas de conocimiento relativas a su zona de interés social, mediado a su vez por la motivación que el individuo tenga hacia el conocimiento. Sin embargo, esta inclinación es en realidad ingenua. La realidad no es que el mundo de lo natural, lo cultural y lo social se dé en la totalidad de la experiencia subjetiva, de tal manera que el sujeto pueda dominar su experiencia en términos de su acción o pensamiento; sino más bien el mundo ya está esbozado y articulado a priori, en estratos de diferente alcance social, lo que implica que el sujeto siempre experimentará cierta situación dentro de un marco ya definido.

En otras palabras, toda interpretación de la experiencia del sujeto dependerá del marco ontológico dado por el grupo social al que pertenece. En este sentido, si tenemos que el sistema educativo es uno de los mecanismos que por excelencia proporciona a los individuos un marco interpretativo de la realidad, podemos notar que en el contexto chileno ese marco interpretativo dado a los estudiantes estará segmentando la posibilidad de comprender la realidad social, según la pertenencia de los niños, las niñas y adolescentes a diferentes grupos socioeconómicos, en consecuencia que podría configurarse como un mecanismo democrático de transmisión del saber cultural y social del mundo. Sobre esta característica reproductiva de diferencias sociales, revisaremos precisamente el aporte de la Teoría de la Reproducción de Bourdieu.

\section{La teoría de la reproducción}

En principio se asumía que el sistema escolar gozaba de autonomía relativa, según la cual la enseñanza sirve insustituiblemente a la definición de estructuras sociales. Así, la reproducción de relaciones de clases en la escuela resulta de la acción pedagógica ejercida sobre los sujetos, que por lo demás ya han recibido acciones pedagógicas en el ámbito de su educación primaria (que incluiría cierto capital cultural y cierta postura frente a la cultura) (Bourdieu ${ }^{3}$ y Passeron, 1996).

Desde este supuesto, cada acción pedagógica ejercida en la escuela tendrá una eficacia distinta en cada individuo, dependiendo de las

3 Escritor y sociólogo francés (1930-2002). Licenciado en Filosofía, uno de los padres de la sociología contemporánea. Fue vicepresidente del Parlamento Internacional de Escritores, entidad dedicada a proteger a los escritores e intelectuales víctimas de persecución por razones ideológicas o religiosas (Fuente: www.mcnbiografias.com). 
caracterizaciones culturales y sociales que le preexistan, de tal manera que en el momento en que la escuela evalúa los resultados diferenciados de los estudiantes frente a las acciones pedagógicas, como si solamente respondiesen a la acción propiamente tal, estará contribuyendo a reproducir y legitimar la estratificación social, persuadiendo a los estudiantes de que estas diferencias son naturales, no sociales (Bourdieu y Passeron, 1996).

Por otra parte, la especificación de formas y productos derivados de una acción pedagógica ejercida en el ámbito escolar reproduce también, cierta cultura dominante de la enseñanza, reservándose el monopolio de la violencia simbólica legitimada, expresada en la imposición y la inculcación arbitraria de un modelo cultural (por ejemplo, la estructura familiar, o la posición de la familia en la estructura social) (Bourdieu y Passeron, 1996).

De acuerdo con Bourdieu (1994), las clases sociales corresponden a posiciones separadas por distancias relativas, a cuyos agentes se les confiere fuerza, poder y beneficios derivados del espacio social y condiciones de existencia comunes. Así, la relación entre el habitus y las probabilidades otorgadas objetivamente, por la sociedad definirán la práctica del agente en su posición y espacio.

Una de las nociones más comunes en el desarrollo sociológico del siglo XX, es el habitus, que es transmitido desde cada espacio social, de modo que cada agente interiorice ciertas prácticas que lo legitiman simbólica y materialmente en dicho espacio (CESCC-OPECH, 2009), configurándose como el fundamento de la homogeneización y la predictibilidad de los comportamientos de un grupo (Bourdieu, 1994). Es a través de la socialización primaria donde, cada agente incorpora un conjunto de disposiciones duraderas y transferibles, generativas de todas las prácticas, los esquemas, las percepciones y los comportamientos propios de la clase (Cervini y Gosende, 2008). En definitiva, habitus es el modo en que las personas operan en el mundo, tanto de forma representacional como emocional y corporalmente, inscribiéndose en el cuerpo y en la mente de los individuos, quitando, así, la misma individualidad y transformándolos en agentes que tienen y utilizan sus modos de representación en los campos en que se desenvuelven (Aedo, 2014).

De acuerdo con el grupo de investigación CESCC-OPECH (2009), Bourdieu aporta algunas precisiones sobre la relación entre el habitus de clase y el individual. Al respecto, el autor precisa que esto 
no es el reflejo de una clase, sino más bien un sistema subjetivo, pero no individual, de estructuras interiorizadas. Así, en el plano individual, cada habitus es una variante estructural de los otros individuales.

De acuerdo con Aedo (2014), un punto elemental respecto del habitus de Bourdieu es que no hay agente sin habitus, ni hay habitus sin campo. Un agente puede cambiar de posición dentro de un campo, o cambiar de campo incluso, pero mantendrá su mismo habitus. Una posibilidad excepcional, sin embargo, en que el habitus puede variar, está la movilidad social.

La movilidad social se entiende como el ascenso o el descenso de los agentes dentro de las posiciones de espacio social, para lo cual se requiere acumular o perder, respectivamente, el capital que soporta esa posición. Es la experiencia, tomada por Bourdieu de la sociología fenomenológica, la que rompe el estructuralismo, introduciendo a un sujeto experimentador que es un producto histórico y social, abriendo así, la posibilidad, aunque escasa, de que el habitus pierda su capacidad práctica, ya sea porque las expectativas esperadas no se realizan, o bien por la realización de expectativas imposibles (Aedo, 2014).

\section{Aplicaciones en educación}

\section{Educación escolar}

La teoría del habitus señala a la escuela como una fuerza formadora de habitus, contribuyendo a la creación de esquemas particulares, aplicables en determinados campos del pensamiento y de la acción. En este sentido, la escuela demanda e impone -fundamentalmente, a través de la enseñanza y la evaluación- el capital cultural a la clase, y reconoce su habitus, sin importar el origen social del estudiante, aumentando la probabilidad de éxito escolar de cada estudiante en tanto, agente de una clase social particular (CESCC-OPECH, 2009).

De acuerdo con Cervini y Gosende (2008), a través del currículum directo la escuela traspasa esquemas de pensamiento y acción particularizados, según asignaturas, mientras que en plano del currículum oculto, enseña "el oficio del estudiante" desde el sentido común, las normas y las actitudes frente a las tareas escolares. Por otra parte, en el ámbito de la socialización primaria y escolar, de acuerdo con Bourdieu (2000), el aprendizaje de los roles asociados al género es más tácito que el de otros saberes. En este sentido, la identidad masculina y la femenina son codificadas mediante principios opuestos de comportamiento y mantención 
del cuerpo, los cuales son naturalizados a través de una ética -y estéticasocial. Por ejemplo, la moral femenina suele imponerse a través de una disciplina concerniente a las partes del cuerpo (presión de las ropas y de las cabelleras) y movimientos asociados a la sumisión (doblar el cuerpo, agacharse, posiciones curvadas) y docilidad. Por otra parte, la moral masculina alude al enfrentamiento (mirarse a la cara, en una postura firme o "militar"), como demostración de rectitud y dominación.

Este dominio del cuerpo permite a los sujetos la adaptación a los juegos sociales concernientes a cada rol. Por ejemplo, la política, los negocios y la ciencia estarían ligados a la virilidad; quedando las mujeres excluidas de los juegos de poder, lo cual sería ampliamente reforzado por la educación primaria. Esta misma lógica de exclusión se perpetuaría hasta determinar el acceso a las diferentes profesiones y a las posiciones en cada una de ellas (Bourdieu, 2000).

\section{Educación superior}

En Chile, es característico que el sistema de enseñanza disimule su "función social" de optimizar las diferencias de clase bajo su función técnica de producción de trabajadores calificados. No cabe duda, señala Bourdieu (2000), que las sociedades modernas consiguen cada vez más que la escuela produzca y garantice un número mayor de individuos calificados, es decir, adaptados cada vez más a las demandas de la economía, pero esta calificación en la práctica no se traduce en una calificación social como "efecto de certificación". Esto explica que existan tantos puestos profesionales ocupados con distintos títulos y remuneraciones desiguales, por individuos que solo se diferencian por el nivel educacional alcanzado, y no por lo indispensables que puedan ser en su eficacia técnica, como es propio, por ejemplo, de algunas carreras técnico-profesionales.

Por último, es posible señalar que el examen universitario es una de las expresiones más visibles de la dominación sociocultural, al imponer una sanción frente a la definición social del saber e inclusive, de la manera de expresarlo. En otras palabras, los exámenes son un mecanismo jurídico de regulación de la transmisión de los saberes culturales dominantes (Bourdieu, 2000).

En Chile, diversas investigaciones han constatado que la Prueba de Selección Universitaria (PSU) es uno de los factores más incidentes en las inequidades del acceso a la educación superior, al enfrentar 
por igual a estudiantes que han accedido a conocimientos del mundo social en mayor profundidad y calidad que otros, incluso en términos del espacio social de los estudiantes (Miranda, 2017). Un ejemplo de programa orientado a reducir esta diferencia es el propedéutico, cuya finalidad es promover a los alumnos con mayores logros educativos en contextos de riesgo social con independencia de su puntaje PSU. No obstante, los autores (CESCC-OPECH, 2009) consideran que es necesario ir más allá de la lógica meritocrática para superar las brechas sociales, siendo más pertinente indagar en el fundamento político que soporta la desigualdad.

\section{Conclusiones}

Tradicionalmente, se ha sostenido que la educación es el principal vehículo de movilidad e inclusión social, desde que se concibe en la modernidad como la materialización de un pacto social, en el que las familias conceden al Estado la responsabilidad sobre la formación de sus hijos, acabando progresivamente con las diferencias de clase (Vain, 2009).

Sin embargo, los estado-nación han transitado a través de distintos modelos de desarrollo que a su vez han impactado en la configuración del sistema educativo, pasando por el estado de bienestar (Puiggros, 2001) y el estado desarrollista (Ruíz, 1994) hasta el estado neoliberal (Villatorio, 2014) -como el caso chileno-, dejando quebrantada la promesa original de movilidad social, mediante la reproducción de formas de segmentación y exclusión, y cerrando a los sectores marginados la posibilidad de ascender en los estratos de la sociedad, limitado por la oferta de distintos niveles de calidad educativa, según cada grupo socioeconómico.

Al respecto, basta citar una revisión de la política educacional chilena por parte de la Organización para la Cooperación y Desarrollo Económico (OCDE, 2004), en que se sostiene respecto del sistema educacional chileno:

El punto importante (es) (...) que el sistema educacional está conscientemente estructurado por clases. (...) la selectividad por escuelas y un énfasis en la "selección" produce un sistema altamente estratificado en el cual hay una creciente concentración de niños en escuelas con antecedentes socio-económicos 
similares. (...) La democracia no se ve favorecida por una estratificación tan intensa (p. 277).

Por lo tanto, el desafío que se desprende, particularmente en contextos de democracia debilitada por la desigualdad social, es el de fortalecer el eslabón educativo a través de un diseño y desarrollo curricular alineado a la formación democrática de los estudiantes, perspectiva que es conocida como educación para la democracia (Cox, Bascopé, Castillo, Miranda, y Bonhomme, 2014), y en este sentido son innumerables los aportes que las ciencias sociales han desarrollado, para la comprensión de las problemáticas sociales, como las derivadas del sistema educativo chileno y sus deficiencias.

Esta breve reflexión muestra a la sociología como uno de los ángulos de observación privilegiados, para dar luces sobre este fenómeno, quedando el desafío, por una parte, de ampliar e integrar estos marcos interpretativos, con el aporte de otras disciplinas, y por otra parte, de fortalecer el vínculo entre la producción de conocimiento científico y el desarrollo de políticas sociales, a fin de cumplir la promesa, en materia educativa, de una educación para la paz y la igualdad como valores fundamentales de una sociedad democrática.

\section{Referencias}

Aedo, A. (2014). El habitus y la movilidad social: de la modificación del sistema de disposiciones a la transformación de la estructura de clases. Revista de Sociología, 29, 57-75.

Bourdieu, P. y Passeron, J. C. (1996). La reproducción: elementos para una teoría del sistema de enseñanza. México D. F.: Editorial Laia.

Bourdieu, P. (1994). ¿Qué es lo que hace una clase social? Acerca de la existencia teórica y práctica de los grupos. Revista Paraguaya de Sociología, 31(89), 7-21.

Bourdieu, P. (2000). La dominación masculina. Barcelona: Anagrama.

Cervini, R. y Gosende, E. (2008). Características no cognitivas, habitus y progreso del aprendizaje de matemática en la educación general básica (EGB) de la provincia de Buenos Aires (República Argentina). Interdisciplinaria, 25(2), 143-179.

CESCC-OPECH (2009). Acceso a la educación superior: el mérito y la (re)producción de la desigualdad. Observatorio chileno de 
políticas educativas. Recuperado de http://www.opech.cl/inv/ analisis/acceso.pdf

Cox, C., Bascopé, M., Castillo, J. C., Miranda, D. y Bonhomme, M. (2014). Educación ciudadana en América Latina: prioridades de los currículums escolares. IBE Working Papers on Curriculum Issues, 14, 1-41.

García-Huidobro, J. (2013). Educación inclusiva y democracia. En F. Migeon (Presidencia), Colloque international L'éducation inclusive: Une formation á inventer, Coloquio llevado a cabo en París, Francia.

Miranda, F. (2017). Análisis del comportamiento espacial de los resultados PSU (proceso de admisión 2016). Revista Centro de Investigación Social, 22, 63-85.

OCDE (2004). Revisión de políticas nacionales de educación: educación terciaria en Chile. Santiago de Chile: OCDE Ediciones.

Puiggros, A. (2001). Educación y poder: los desafíos del próximo siglo. En Carlos Torres (Ed.). Paulo Freire y la agenda de la educación latinoamericana en el siglo XXI. Buenos Aires: CLACSO.

Rizo, M. (2006). La interacción y la comunicación desde los enfoques de la psicología social y la sociología fenomenológica. Análisi: Quaderns de Comunicació i Cultura, 33, 45-62.

Rizo, M. (2014). De lo interpersonal a lo intersubjetivo. Algunas claves teóricas y conceptuales para definir la comunicación intersubjetiva. Quórum Académico, 11(2), 290-307.

Ruíz, C. (1994). Educación, desarrollo y modernización. Revista de Sociología, 0(9), 83-93.

Schütz, A. (1970). Collected Papers III. Studies in Phenomenological Philosophy. The Hague: Springer Netherlands.

Vain, P. D. (2009). Escuela, estado y familia. Un pacto por redefinir. Educación, Lenguaje y Sociedad, 6(6), 329-344.

Villalobos, C. y Valenzuela, J. P. (2012). Polarización y cohesión social del sistema escolar chileno. Revista de Análisis Económico, 27(2), 145-172.

Villatorio, A. (2014). Globalización: crisis, educación y democracia en México (2000-2012). Cuadernos Intercambio sobre Centroamérica y el Caribe, 11(1), 205-224. 
